\title{
POINTWISE SIMULTANEOUS CONVERGENCE OF EXTENDED LAGRANGE INTERPOLATION WITH ADDITIONAL KNOTS
}

\author{
GIULIANA CRISCUOLO, GIUSEPPE MASTROIANNI, AND PÉTER VÉRTESI
}

\begin{abstract}
In numerical analysis it is important to construct interpolating polynomials approximating a given function and its derivatives simultaneously. The authors define some new good interpolating matrices with "many" nodes close to the endpoints of the interval and also give error estimates.
\end{abstract}

\section{INTRODUCTION}

In numerical analysis it is important to construct interpolating polynomials approximating a given function and its derivatives simultaneously. For this reason we try to define new interpolating matrices with nodes easily calculable and "small" Lebesgue functions or constants.

Very recently, the weights $w_{1}(x)=(1-x) w(x), w_{2}(x)=(1+x) w(x)$, and $w_{3}(x)=\left(1-x^{2}\right) w(x)$ with $w \in G J$ (cf. (2.8)), and the roots of the corresponding orthonormal polynomials $\left\{p_{m}(w)\right\}$ and $\left\{p_{m}\left(w_{i}\right)\right\} \quad(i=1,2,3)$ were considered in [2]. As it turned out, all the zeros of $q_{2 m}=p_{m}\left(w_{1}\right) p_{m}\left(w_{2}\right)$ are simple; a similar result holds for $\bar{q}_{2 m+1}=p_{m+1}(w) p_{m}\left(w_{3}\right)$. (See [2, Theorems 2.2 and 2.3].) These facts allow us to define two so-called extended interpolatory matrices $X_{1}$ and $X_{2}$ having as nodes the zeros of $q_{2 m}$ and $\bar{q}_{2 m+1}$, respectively, and to consider uniform convergence of the corresponding Lagrange interpolation. (See [2, Theorems 4.1 and 4.3].)

Extended interpolatory matrices were used for numerical quadrature (Kronrod formula) and for the numerical solution of singular integral equations by several authors. The interested reader may consult the exhaustive survey papers of Gautschi [5] and Monegato [6].

The main goal of the present paper is to achieve good simultaneous approximation of a given function and its derivatives, using the above matrices $X_{1}$ and $X_{2}$ and some additional nodes near the endpoints \pm 1 .

\section{Preliminaries}

Lagrange interpolation. Let $\left\{Q_{n}\right\}$ be a sequence of polynomials $\left(Q_{n} \in \mathbb{P}_{n}\right)$ with zeros $t_{j, n}, j=1,2, \ldots, n$, satisfying

$$
-1<t_{1, n}<t_{2, n}<\cdots<t_{n, n}<1
$$

Received by the editor July 20, 1990.

1991 Mathematics Subject Classification. Primary 41 A05.

This material is based upon work supported by the Italian Research Council (first and second authors), by the Ministero della Ricerca Scientifica (second author), and by Hungarian National Foundation for Scientific Research Grant No. 1801 (third author). 
and

$$
1+t_{1, n} \sim n^{-2} \sim 1-t_{n, n}, \quad n \in N^{1}
$$

Along with the matrix $T=\left\{t_{j, n}, j=1,2, \ldots, n\right\}_{n=1}^{\infty}$ we consider additional matrices $Y=\left\{y_{j, n}, j=1,2, \ldots, s\right\}_{n=1}^{\infty}, Z=\left\{z_{j, n}, j=1,2, \ldots, r\right\}_{n=1}^{\infty}$, $r, s \geq 0$, where

$$
\begin{gathered}
-1 \leq y_{1, n} \leq y_{2, n} \leq \cdots \leq y_{s, n}<t_{1, n}<t_{n, n}<z_{1, n} \leq z_{2, n} \leq \cdots \leq z_{r, n} \leq 1, \\
t_{1, n}-y_{s, n} \sim n^{-2} \sim z_{1, n}-t_{n, n}, \quad n \in N .
\end{gathered}
$$

Moreover, we define the polynomials

$$
\begin{array}{lll}
A_{0}(x) \equiv 1, & A_{s}(x)=\prod_{j=1}^{s}\left(x-y_{j, n}\right), & s>0, \\
B_{0}(x) \equiv 1, & B_{r}(x)=\prod_{j=1}^{r}\left(x-z_{j, n}\right), & r>0 .
\end{array}
$$

For a given matrix $V$ and bounded function $f$, denote by $L_{k}(V ; f)$ the corresponding Lagrange (Hermite) polynomial of degree $k-1$. The polynomial $L_{n+r+s}(X ; f)$ of degree $n+r+s-1$ based on $X=T \cup Y \cup Z$ is

$$
\begin{aligned}
L_{n+r+s}(X ; f)= & A_{s} B_{r} L_{n}\left(T ; \frac{f}{A_{s} B_{r}}\right)+A_{s} Q_{n} L_{r}\left(Z ; \frac{f}{A_{s} Q_{n}}\right) \\
& +B_{r} Q_{n} L_{s}\left(Y ; \frac{f}{B_{r} Q_{n}}\right)
\end{aligned}
$$

where

$$
\begin{aligned}
& L_{n}\left(T ; \frac{f}{A_{s} B_{r}} ; x\right)=\sum_{i=1}^{n} \frac{Q_{n}(x)}{Q_{n}^{\prime}\left(t_{i, n}\right) A_{s}\left(t_{i, n}\right) B_{r}\left(t_{i, n}\right)\left(x-t_{i, n}\right)}, \\
& L_{r}\left(Z ; \frac{f}{A_{s} Q_{n}} ; x\right)=\frac{f\left(z_{1, n}\right)}{A_{s}\left(z_{1, n}\right) Q_{n}\left(z_{1, n}\right)} \\
& +\sum_{i=2}^{r}\left(x-z_{1, n}\right)\left(x-z_{2, n}\right) \cdots\left(x-z_{i-1, n}\right) \\
& \times\left[z_{1, n}, z_{2, n}, \ldots, z_{i, n} ; \frac{f}{A_{s} Q_{n}}\right], \\
& L_{s}\left(Y ; \frac{f}{B_{r} Q_{n}} ; x\right)=\frac{f\left(y_{1, n}\right)}{B_{r}\left(y_{1, n}\right) Q_{n}\left(y_{1, n}\right)} \\
& +\sum_{i=2}^{s}\left(x-y_{1, n}\right)\left(x-y_{2, n}\right) \cdots\left(x-y_{i-1, n}\right) \\
& \times\left[y_{1, n}, y_{2, n}, \ldots, y_{i, n} ; \frac{f}{B_{r} Q_{n}}\right] .
\end{aligned}
$$

${ }^{1}$ If $A$ and $B$ are two expressions depending on some variables, then we write $A \sim B$ if and only if $\left|A B^{-1}\right| \leq$ const and $\left|A^{-1} B\right| \leq$ const ,

uniformly for the variables in question. 
Here, $\left[u_{1}, u_{2}, \ldots, u_{p} ; g\right]$ is the divided difference of the function $g$ at the points $u_{1}, u_{2}, \ldots, u_{p}$. If the function $f$ is not differentiable, then we assume that all the knots are different. When $f \in C^{q}, q \geq 1$, then the multiplicity of each additional knot may be at most $q$, so we may have Hermite interpolation.

In the case $r=0$ we set $L_{r} \equiv 0$, and similarly, if $s=0$, then $L_{s} \equiv 0$. Obviously, when $r=s=0$ the right side of $(2.3)$ becomes $L_{n}(T ; f)$.

Special weights. Let $v^{\gamma, \delta}$ be the Jacobi weight function

$$
v^{\gamma, \delta}(x)=\left\{\begin{array}{ll}
(1-x)^{\gamma}(1+x)^{\delta} & \text { if }|x| \leq 1, \\
0 & \text { if }|x|>1,
\end{array} \quad \gamma, \delta \in \mathbb{R} .\right.
$$

We consider the generalized Jacobi weight $w \quad(w \in \mathrm{GJ})$ defined as follows:

$$
w(x)=\varphi(x) v^{\alpha, \beta}(x), \quad \alpha, \beta>-1,
$$

where the modulus of continuity $\omega(\varphi ;)$ of the function $\varphi>0$ satisfies $\int_{0}^{1} \omega(\varphi ; t) t^{-1} d t<\infty$.

Then, let $\left\{p_{m}(w)\right\}$ be the system of orthonormal polynomials corresponding to the weight function $w \in \mathrm{GJ}$, i.e.,

$$
p_{m}(w ; x)=\alpha_{m}(w) x^{m}+\text { lower-degree terms }, \quad \alpha_{m}(w)>0,
$$

and

$$
\int_{-1}^{1} p_{m}(w ; x) p_{n}(w ; x) w(x) d x=\delta_{m, n} .
$$

We denote by $x_{i, m}=x_{i, m}(w), i=1,2, \ldots, m$, the zeros of $p_{m}(w)$, with

$$
-1<x_{1, m}<x_{2, m}<\cdots<x_{m, m}<1 \text {, }
$$

and by $\lambda_{i, m}(w)=\lambda_{m}\left(w ; x_{i, m}(w)\right), i=1,2, \ldots, m$, the Christoffel constants, where

$$
\lambda_{m}(w ; x)=\left[\sum_{i=0}^{m-1} p_{i}^{2}(w ; x)\right]^{-1}
$$

is the $m$ th Christoffel function.

Extended Lagrange interpolation. Let $w \in G J$ be defined by (2.8). We consider the weight functions

$$
\begin{aligned}
& w_{1}(x)=w(x)(1-x), \\
& w_{2}(x)=w(x)(1+x), \\
& w_{3}(x)=w(x)\left(1-x^{2}\right),
\end{aligned}
$$

and the corresponding systems of orthonormal polynomials

$$
\left\{p_{m}\left(w_{1}\right)\right\}, \quad\left\{p_{m}\left(w_{2}\right)\right\}, \quad\left\{p_{m}\left(w_{3}\right)\right\} .
$$
i.e.,

It is known [2] that the zeros $x_{i, m}\left(w_{3}\right)$ interlace with the zeros $x_{i, m+1}(w)$,

$$
x_{i, m+1}(w)<x_{i, m}\left(w_{3}\right)<x_{i+1, m+1}(w), \quad i=1, \ldots, m, m \in N .
$$

In [2] it is also proved that the polynomials $p_{m}\left(w_{1}\right)$ and $p_{m}\left(w_{2}\right)$ have no common zeros; further,

$$
x_{i, m}\left(w_{1}\right)<x_{i, m}\left(w_{2}\right), \quad i=1, \ldots, m, m \in N .
$$


Therefore, the zeros of $p_{m+1}(w) p_{m}\left(w_{3}\right)$ and those of $p_{m}\left(w_{1}\right) p_{m}\left(w_{2}\right)$ satisfy conditions (2.1) and (2.2).

Moreover, we recall a more precise result on the distribution of the zeros of $p_{m+1}(w) p_{m}\left(w_{3}\right)$ and $p_{m}\left(w_{1}\right) p_{m}\left(w_{2}\right)$ :

$$
x_{i, m}\left(w_{3}\right)-x_{i, m+1}(w) \sim \frac{\sqrt{1-x^{2}}}{m} \sim x_{i+1, m+1}(w)-x_{i, m}\left(w_{3}\right),
$$

uniformly in $1 \leq i \leq m, m \in N$, and $x \in\left[x_{i, m+1}(w), x_{i+1, m+1}(w)\right]$,

$$
x_{i, m}\left(w_{2}\right)-x_{i, m}\left(w_{1}\right) \sim \frac{\sqrt{1-x^{2}}}{m} \sim x_{i+1, m}\left(w_{1}\right)-x_{i, m}\left(w_{2}\right),
$$

uniformly in $1 \leq i \leq m-1, m \in N$, and $x \in\left[x_{i, m}\left(w_{1}\right), x_{i+1, m}\left(w_{1}\right)\right]$. (See $[2$, Theorems 3.1, 3.2].)

Now, for a bounded function $f$, we define the "extended Lagrange interpolating polynomial" $L_{2 m+1, r, s}\left(w, w_{3} ; f\right)$ as the Lagrange (Hermite) polynomial based on the zeros of $p_{m+1}(w) p_{m}\left(w_{3}\right)$ and on the knots of the matrix $Y \cup Z$. Replacing $Q_{n}$ by $p_{m+1}(w) p_{m}\left(w_{3}\right)$ in $(2.3)$, we get

$$
\begin{aligned}
& L_{2 m+1, r, s}\left(w, w_{3} ; f ; x\right) \\
& =A_{s}(x) B_{r}(x) p_{m+1}(w ; x) p_{m}\left(w_{3} ; x\right) \\
& \quad \times\left\{\sum_{i=1}^{m+1} \frac{f\left(x_{i, m+1}(w)\right) A_{s}^{-1}\left(x_{i, m+1}(w)\right) B_{r}^{-1}\left(x_{i, m+1}(w)\right)}{p_{m+1}^{\prime}\left(w ; x_{i, m+1}(w)\right) p_{m}\left(w_{3} ; x_{i, m+1}(w)\right)\left(x-x_{i, m+1}(w)\right)}\right. \\
& \left.\quad+\sum_{i=1}^{m} \frac{f\left(x_{i, m}\left(w_{3}\right)\right) A_{s}^{-1}\left(x_{i, m}\left(w_{3}\right)\right) B_{r}^{-1}\left(x_{i, m}\left(w_{3}\right)\right)}{p_{m+1}\left(w ; x_{i, m}\left(w_{3}\right)\right) p_{m}^{\prime}\left(w_{3} ; x_{i, m}\left(w_{3}\right)\right)\left(x-x_{i, m}\left(w_{3}\right)\right)}\right\} \\
& \quad+A_{s}(x) L_{r}\left(Z ; \frac{f}{A_{s} p_{m+1}(w) p_{m}\left(w_{3}\right)} ; x\right) \\
& +B_{r}(x) L_{s}\left(Y ; \frac{f}{B_{r} p_{m+1}(w) p_{m}\left(w_{3}\right)} ; x\right) .
\end{aligned}
$$

Recalling that

$$
\begin{aligned}
& p_{m+1}^{\prime}\left(w ; x_{i, m+1}(w)\right) p_{m}\left(w_{3} ; x_{i, m+1}(w)\right) \\
& \quad=C_{m} \lambda_{i, m+1}^{-1}(w)\left(1-x_{i, m+1}^{2}(w)\right)^{-1}, \quad i=1, \ldots, m+1, m \in N,
\end{aligned}
$$

and

$$
\begin{aligned}
& p_{m+1}\left(w ; x_{i, m}\left(w_{3}\right)\right) p_{m}^{\prime}\left(w_{3} ; x_{i, m}\left(w_{3}\right)\right) \\
& \quad=-C_{m} \lambda_{i, m}^{-1}\left(w_{3}\right), \quad i=1, \ldots, m, m \in N,
\end{aligned}
$$

where $C_{m}=\alpha_{m}\left(w_{3}\right) \alpha_{m+1}^{-1}(w)+\alpha_{m+1}(w) \alpha_{m}^{-1}\left(w_{3}\right)<\infty$ (see [2, Theorem 2.2]), and introducing the notation

$$
H_{m}(w ; f ; x)=\sum_{i=1}^{m} \frac{\lambda_{i, m}(w)}{x-x_{i, m}(w)} f\left(x_{i, m}(w)\right),
$$


we can write

$$
\begin{aligned}
& L_{2 m+1, r, s}\left(w, w_{3} ; f\right) \\
& =p_{m+1}(w) p_{m}\left(w_{3}\right) \\
& \quad \times\left\{C_{m}^{-1} A_{s} B_{r}\left[H_{m+1}\left(w ; v^{1,1} \frac{f}{A_{s} B_{r}}\right)-H_{m}\left(w_{3} ; \frac{f}{A_{s} B_{r}}\right)\right]\right. \\
& \quad+A_{s} L_{r}\left(Z ; \frac{f}{A_{s} p_{m+1}(w) p_{m}\left(w_{3}\right)}\right) \\
& \left.\quad+B_{r} L_{s}\left(Y ; \frac{f}{B_{r} p_{m+1}(w) p_{m}\left(w_{3}\right)}\right)\right\} .
\end{aligned}
$$

Similarly, we define the "extended Lagrange interpolating polynomial" $L_{2 m, r, s}\left(w_{1}, w_{2} ; f\right)$ on the zeros of $p_{m}\left(w_{1}\right) p_{m}\left(w_{2}\right)$ and on the knots of the matrix $Y \cup Z$ by

$$
\begin{aligned}
& L_{2 m, r, s}\left(w_{1}, w_{2} ; f\right) \\
& =p_{m}\left(w_{1}\right) p_{m}\left(w_{2}\right) \\
& \quad \times\left\{D_{m}^{-1} A_{s} B_{r}\left[H_{m}\left(w_{2} ; v^{1,0} \frac{f}{A_{s} B_{r}}\right)-H_{m}\left(w_{1} ; v^{0,1} \frac{f}{A_{s} B_{r}}\right)\right]\right. \\
& \quad+A_{s} L_{r}\left(Z ; \frac{f}{A_{s} p_{m}\left(w_{1}\right) p_{m}\left(w_{2}\right)}\right) \\
& \left.\quad+B_{r} L_{s}\left(Y ; \frac{f}{B_{r} p_{m}\left(w_{1}\right) p_{m}\left(w_{2}\right)}\right)\right\},
\end{aligned}
$$

with $D_{m}=\alpha_{m}\left(w_{1}\right) / \alpha_{m}\left(w_{2}\right)+\alpha_{m}\left(w_{2}\right) / \alpha_{m}\left(w_{1}\right)<\infty$. (See [2, formula (2.17)].) Finally, recalling that the zeros of the polynomial $p_{m}(w) p_{m+1}(w)$ obviously satisfy conditions (2.1) and (2.2), we can also consider the "extended Lagrange interpolating polynomial" $L_{2 m+1, r, s}(w, w ; f)$ on these zeros and on the knots of the matrix $Y \cup Z$. Thus,

$$
\begin{aligned}
& L_{2 m+1, r, s}(w, w ; f) \\
& =p_{m}(w) p_{m+1}(w) \\
& \quad \times\left\{\frac{\alpha_{m}(w)}{\alpha_{m+1}(w)} A_{s} B_{r}\left[H_{m+1}\left(w ; \frac{f}{A_{s} B_{r}}\right)-H_{m}\left(w ; \frac{f}{A_{s} B_{r}}\right)\right]\right. \\
& \quad+A_{s} L_{r}\left(Z ; \frac{f}{A_{s} p_{m}(w) p_{m+1}(w)}\right) \\
& \left.\quad+B_{r} L_{s}\left(Y ; \frac{f}{B_{r} p_{m}(w) p_{m+1}(w)}\right)\right\} .
\end{aligned}
$$

Let us remark that the zeros of $p_{m}(w) p_{m+1}(w)$ satisfy

$$
\min _{k \in\{i, i+1\}}\left|x_{i, m}(w)-x_{k, m+1}(w)\right| \sim \frac{1-x_{i, m}^{2}(w)}{m},
$$

uniformly in $1 \leq i \leq m, m \in N$, and $x \in\left[x_{i, m+1}(w), x_{i+1, m+1}(w)\right]$. Comparing (2.17) with (2.12) and (2.13), we deduce that the distribution of the zeros of $p_{m}(w) p_{m+1}(w)$ is different from those of $p_{m+1}(w) p_{m}\left(w_{3}\right)$ and $p_{m}\left(w_{1}\right) p_{m}\left(w_{2}\right)$. 
This fact causes the different behavior of the corresponding processes (cf. Theorems 3.1 and 3.2).

\section{CONVERGENCE OF THE EXTENDED INTERPOLATION FORMULAE}

We define the space of functions $C^{q}$ on the interval $[-1,1]$ in the usual way; thus, $f \in C^{q}$ if and only if $f$ is continuous with its derivatives $f^{(j)}$, $j \leq q$, on $[-1,1]$. Furthermore, let $\operatorname{Lip}_{M} \lambda, 0<\lambda \leq 1$, be the class of Hölder-continuous functions, i.e, $f \in \operatorname{Lip}_{M} \lambda$ if and only if $f$ is continuous and its modulus of continuity $\omega(f ;)$ satisfies $\omega(f ; \delta) \leq M \delta^{\lambda}, M \in \mathbb{R}^{+}$.

Theorem 3.1. Let $w, w_{1}, w_{2}, w_{3} \in \mathrm{GJ}$ be the weight functions defined by (2.8)(2.11). Let $f \in C^{q}, q \geq 0$, and let $l \in\{0,1, \ldots, q\}$. Then,

$$
\begin{aligned}
& \left.\begin{array}{l}
\left|\left[f^{(h)}(x)-L_{2 m+1, r, s}^{(h)}\left(w, w_{3} ; f ; x\right)\right]\right| \\
\left|\left[f^{(h)}(x)-L_{2 m, r, s}^{(h)}\left(w_{1}, w_{2} ; f ; x\right)\right]\right|
\end{array}\right\} \\
& \quad \leq \text { const }\left(\frac{\sqrt{1-x^{2}}}{m}+\frac{1}{m^{2}}\right)^{l-h} \omega\left(f^{(q)} ; \frac{1}{m}\right) \frac{\log m}{m^{q-l}}, \\
& |x| \leq 1, h=0,1, \ldots, l,
\end{aligned}
$$

with some constant independent of $f$ and $m$, whenever the integers $r$ and $s$ fulfill

$$
\begin{aligned}
& \frac{l}{2}+\alpha+1 \leq r<\frac{l}{2}+\alpha+2, \\
& \frac{l}{2}+\beta+1 \leq s<\frac{l}{2}+\beta+2 .
\end{aligned}
$$

Theorem 3.2. Let $w \in \mathrm{GJ}$ be the weight function defined by (2.8). Let $f \in C^{q}$, $q \geq 0$, and let $l \in\{0,1, \ldots, q\}$. Then,

$$
\begin{array}{r}
\left|\left[f^{(h)}(x)-L_{2 m+1, r, s}^{(h)}(w, w ; f ; x)\right]\left(\sqrt{1-x^{2}}+\frac{1}{m}\right)\right| \\
\leq \text { const }\left(\frac{\sqrt{1-x^{2}}}{m}+\frac{1}{m^{2}}\right)^{l-h} \omega\left(f^{(q)} ; \frac{1}{m}\right) \frac{\log m}{m^{q-l}}, \\
|x| \leq 1, h=0,1, \ldots, l,
\end{array}
$$

with some constant independent of $f$ and $m$, whenever the integers $r$ and $s$ fulfill

$$
\begin{aligned}
& \frac{l}{2}+\alpha \leq r<\frac{l}{2}+\alpha+1, \\
& \frac{l}{2}+\beta \leq s<\frac{l}{2}+\beta+1 .
\end{aligned}
$$

To complete the previous theorems, we notice that the polynomial $L_{2 m+1}\left(w, w_{3} ; f\right)$ interpolating $f$ only on the zeros of $p_{m+1}(w) p_{m}\left(w_{3}\right)$ generates an error given in [2]. Therefore, by the Gopengauz theorem and Markov's inequality, there follows

$$
\left|f^{(h)}(x)-L_{2 m+1}^{(h)}\left(w, w_{3} ; f ; x\right)\right| \leq \text { const } \sigma_{m} \omega\left(f^{(q)} ; m^{-1}\right) \frac{\log m}{m^{q-2 h}},
$$


where $\sigma_{m}=\max \left(m^{2+2 \alpha}, m^{2+2 \beta}\right),|x| \leq 1$, which is weaker than the first inequality of (3.1). An analogous argument can be developed for the Lagrange polynomials corresponding to the zeros of $p_{m}\left(w_{1}\right) p_{m}\left(w_{2}\right)$ and $p_{m}(w) p_{m+1}(w)$. Hence, adding knots near the endpoints \pm 1 seems necessary to obtain good interpolation processes. We recall also that, if $w$ is a Jacobi weight, so are $w_{1}, w_{2}$, and $w_{3}$; therefore, the interpolation knots are computable efficiently.

Finally, we remark that (3.4) (because of the factor $\sqrt{1-x^{2}}+m^{-1}$ ) is weaker than (3.1). The reason probably is the distribution of the zeros (cf. (2.17)).

Inequalities (3.2) and (3.3) can be rewritten as

$$
\begin{aligned}
& r-\frac{l}{2}-2<\alpha \leq r-\frac{l}{2}-1, \\
& s-\frac{l}{2}-2<\beta \leq s-\frac{l}{2}-1,
\end{aligned}
$$

and (3.5) and (3.6) as

$$
\begin{aligned}
& r-\frac{l}{2}-1<\alpha \leq r-\frac{l}{2}, \\
& s-\frac{l}{2}-1<\beta \leq s-\frac{l}{2} .
\end{aligned}
$$

Obviously, since $\alpha, \beta>-1$, inequalities (3.7) and (3.8) imply $r>l / 2$ and $s>l / 2$; similarly, (3.9) and (3.10) imply $r>l / 2-1$ and $s>l / 2-1$. In any case, one can define infinitely many good matrices satisfying (3.7) and (3.8) ((3.9) and (3.10)) and the above condition for which (3.1) ((3.4)) holds true.

When the additional knots coincide with -1 and 1 , as we have already observed in $\S 2$, we obtain a Hermite interpolation process $(r, s \leq q+1)$. Further, (3.1) and (3.4) can then be improved.

Theorem 3.3. Let $f \in C^{q}, q \geq 0$, and let $l \in\{0,1, \ldots, q\}$. Assume that the values $f^{(i)}(-1), i=0,1, \ldots, s-1$, and $f^{(i)}(1), i=0,1, \ldots, r-1$, with $r, s \leq q+1$, are known, and let

$$
y_{i, m}=-1, \quad i=1,2, \ldots, s, \quad z_{i, m}=1, \quad i=1,2, \ldots, r .
$$

If $r>l / 2, s>l / 2$, and $\alpha$ and $\beta$ satisfy (3.7) and (3.8), then

$$
\begin{aligned}
& \left.\begin{array}{l}
\left|\left[f^{(h)}(x)-L_{2 m+1, r, s}^{(h)}\left(w, w_{3} ; f ; x\right)\right]\right| \\
\left|\left[f^{(h)}(x)-L_{2 m, r, s}^{(h)}\left(w_{1}, w_{2} ; f ; x\right)\right]\right|
\end{array}\right\} \\
& \leq \text { const }\left(\frac{\sqrt{1-x^{2}}}{m}\right)^{l-h} \omega\left(f^{(q)} ; \frac{1}{m}\right) \frac{\log m}{m^{q-l}}, \\
& |x| \leq 1, h=0,1, \ldots, l,
\end{aligned}
$$

with some constant independent of $f$ and $m$.

Theorem 3.4. Let $f \in C^{q}, q \geq 0$, and let $l \in\{0,1, \ldots, q\}$. Assume that the values $f^{(i)}(-1), i=0,1, \ldots, s-1$, and $f^{(i)}(1), i=0,1, \ldots, r-1$, with $r, s \leq q+1$, are known, and let (3.11) hold. If $r>l / 2-1, s>l / 2-1$, and 
$\alpha$ and $\beta$ satisfy (3.9) and (3.10), then

$$
\begin{array}{r}
\left|\left[f^{(h)}(x)-L_{2 m+1, r, s}^{(h)}(w, w ; f ; x)\right] \sqrt{1-x^{2}}\right| \\
\leq \text { const }\left(\frac{\sqrt{1-x^{2}}}{m}\right)^{l-h} \omega\left(f^{(q)} ; \frac{1}{m}\right) \frac{\log m}{m^{q-l}}, \\
|x| \leq 1, h=0,1, \ldots, l,
\end{array}
$$

with some constant independent of $f$ and $m$.

In both theorems we again define infinitely many "good" matrices. In the estimates (3.1), (3.4), (3.12), and (3.13), the ordinary modulus of continuity $\omega\left(f^{(q)} ; 1 / m\right)$ appears. In general, we cannot replace the term $1 / m$ in the modulus of continuity by $\sqrt{1-x^{2}} / m+m^{-2}$ in (3.1) and (3.4), and by $\sqrt{1-x^{2}} / m$ in (3.12) and (3.13). However, if the $q$ th derivative of $f$ is Hölder continuous, i.e., $f^{(q)} \in \operatorname{Lip}_{M} \lambda$, then we can state the following theorems.

Theorem 3.5. Let $w, w_{1}, w_{2}, w_{3} \in \mathrm{GJ}$ be the weight functions defined by (2.8)(2.11). Let $f \in C^{q}, q \geq 0$, and $f^{(q)} \in \operatorname{Lip}_{M} \lambda, 0<\lambda \leq 1$, and let $l \in$ $\{0,1, \ldots, q\}$. Then, for any exponents $\alpha, \beta>-1$, there exist positive integers $r, s$ defined by

such that

$$
\begin{aligned}
& \frac{l+\lambda}{2}+\alpha+1 \leq r<\frac{l+\lambda}{2}+\alpha+2, \\
& \frac{l+\lambda}{2}+\beta+1 \leq s<\frac{l+\lambda}{2}+\beta+2,
\end{aligned}
$$

$$
\begin{aligned}
& \left.\begin{array}{l}
\left|\left[f^{(h)}(x)-L_{2 m+1, r, s}^{(h)}\left(w, w_{3} ; f ; x\right)\right]\right| \\
\left|\left[f^{(h)}(x)-L_{2 m, r, s}^{(h)}\left(w_{1}, w_{2} ; f ; x\right)\right]\right|
\end{array}\right\} \\
& \leq \text { const }\left(\frac{\sqrt{1-x^{2}}}{m}+\frac{1}{m^{2}}\right)^{l+\lambda-h} \frac{\log m}{m^{q-l}}, \\
& |x| \leq 1, h=0,1, \ldots, l,
\end{aligned}
$$

with some constant independent of $f$ and $m$.

Theorem 3.6. Let $w \in \mathrm{GJ}$ be the weight function defined by (2.8). Let $f \in C^{q}$, $q \geq 0$, and $f^{(q)} \in \operatorname{Lip}_{M} \lambda, 0<\lambda \leq 1$, and let $l \in\{0,1, \ldots, q\}$. Then, for any exponents $\alpha, \beta>-1$, there exist positive integers $r, s$ defined by

such that

$$
\begin{aligned}
& \frac{l+\lambda}{2}+\alpha \leq r<\frac{l+\lambda}{2}+\alpha+1, \\
& \frac{l+\lambda}{2}+\beta \leq s<\frac{l+\lambda}{2}+\beta+1,
\end{aligned}
$$

$$
\begin{gathered}
\left|\left[f^{(h)}(x)-L_{2 m+1, r, s}^{(h)}(w, w ; f ; x)\right]\right|\left(\sqrt{1-x^{2}}+\frac{1}{m}\right) \\
\leq \text { const }\left(\frac{\sqrt{1-x^{2}}}{m}+\frac{1}{m^{2}}\right)^{l+\lambda-h} \frac{\log m}{m^{q-l}}, \\
|x| \leq 1, h=0,1, \ldots, l,
\end{gathered}
$$

with some constant independent of $f$ and $m$. 
Theorem 3.7. Let $f \in C^{q}, q \geq 0$, and $f^{(q)} \in \operatorname{Lip}_{M} \lambda, 0<\lambda \leq 1$, and let $l \in\{0,1, \ldots, q\}$. Assume that the values $f^{(i)}(-1), i=0,1, \ldots, s-1$, and $f^{(i)}(1), i=0,1, \ldots, r-1$, with $r, s \leq q+1$, are known, and let (3.11) hold. If $r>(l+\lambda) / 2, s>(l+\lambda) / 2$, and $\alpha$ and $\beta$ satisfy

$$
\begin{aligned}
& r-\frac{l+\lambda}{2}-2<\alpha \leq r-\frac{l+\lambda}{2}-1, \\
& s-\frac{l+\lambda}{2}-2<\beta \leq s-\frac{l+\lambda}{2}-1,
\end{aligned}
$$

then

$$
\begin{aligned}
& \left.\begin{array}{l}
\left|\left[f^{(h)}(x)-L_{2 m+1, r, s}^{(h)}\left(w, w_{3} ; f ; x\right)\right]\right| \\
\left|\left[f^{(h)}(x)-L_{2 m, r, s}^{(h)}\left(w_{1}, w_{2} ; f ; x\right)\right]\right|
\end{array}\right\} \\
& \quad \leq \operatorname{const}\left(\frac{\sqrt{1-x^{2}}}{m}\right)^{l+\lambda-h} \frac{\log m}{m^{q-l}}, \quad|x| \leq 1, h=0,1, \ldots, l,
\end{aligned}
$$

with some constant independent of $f$ and $m$.

Theorem 3.8. Let $f \in C^{q}, q \geq 0$, and $f^{(q)} \in \operatorname{Lip}_{M} \lambda, 0<\lambda \leq 1$, and let $l \in\{0,1, \ldots, q\}$. Assume that the values $f^{(i)}(-1), i=0,1, \ldots, s-1$, and $f^{(i)}(1), i=0,1, \ldots, r-1$, with $r, s \leq q+1$, are known, and let (3.11) hold. If $r>(l+\lambda) / 2-1, s>(l+\lambda) / 2-1$, and $\alpha$ and $\beta$ satisfy

$$
\begin{aligned}
& r-\frac{l+\lambda}{2}-1<\alpha \leq r-\frac{l+\lambda}{2}, \\
& s-\frac{l+\lambda}{2}-1<\beta \leq s-\frac{l+\lambda}{2},
\end{aligned}
$$

then

$$
\begin{array}{r}
\left|\left[f^{(h)}(x)-L_{2 m+1, r, s}^{(h)}(w, w ; f ; x)\right] \sqrt{1-x^{2}}\right| \\
\leq \operatorname{const}\left(\frac{\sqrt{1-x^{2}}}{m}\right)^{l+\lambda-h} \frac{\log m}{m^{q-l}}, \\
|x| \leq 1, h=0,1, \ldots, l,
\end{array}
$$

with some constant independent of $f$ and $m$.

Again, we have infinitely many "good" matrices.

\section{Proofs}

Let

$$
\mu(x)=\varphi(x) v^{\gamma, \delta}(x) \in \mathrm{GJ},
$$

and denote by $x_{i, m}(\mu), i=1,2, \ldots, m$, the zeros of the $m$ th orthonormal polynomial $p_{m}(\mu)$ corresponding to the weight $\mu$.

For convenience, we collect some properties of the generalized Jacobi polynomials $p_{m}(\mu)$ which will be applied in the sequel. Set $x_{i, m}(\mu)=\cos \theta_{i, m}$ for 
$0 \leq i \leq m+1$, where $x_{0, m}(\mu)=-1, x_{m+1, m}(\mu)=1$, and $0 \leq \theta_{i, m} \leq \pi$. Then

$$
\left|\theta_{i, m}-\theta_{i+1, m}\right| \sim m^{-1},
$$

uniformly for $0 \leq i \leq m, m \in N$ [7, Theorem 9.22, p. 166]. We have

$$
\lambda_{i, m}(\mu) \sim m^{-1}\left(1-x_{i, m}(\mu)\right)^{\gamma+1 / 2}\left(1+x_{i, m}(\mu)\right)^{\delta+1 / 2},
$$

uniformly for $1 \leq i \leq m, m \in N$ [7, Theorem 6.3.28, p. 120]. There holds

$$
\left|p_{m}(\mu ; x)\right| \leq \operatorname{const}\left(\sqrt{1-x}+m^{-1}\right)^{-\gamma-1 / 2}\left(\sqrt{1+x}+m^{-1}\right)^{-\delta-1 / 2}
$$

uniformly for $-1 \leq x \leq 1$ and $m \in N$ [1, Theorem 1.1, p. 226]. In particular,

$$
\left|p_{m}(\mu ; x)\right| \sim m^{\gamma+1 / 2} \sim p_{m}(\mu ; 1), \quad 1-m^{-2} \leq x \leq 1,
$$

and

$$
\left|p_{m}(\mu ; x)\right| \sim m^{\delta+1 / 2} \sim\left|p_{m}(\mu ;-1)\right|, \quad-1 \leq x \leq-1+m^{-2},
$$

uniformly for $m \in N$ (see also [8]). Furthermore,

$$
\begin{aligned}
\left|p_{m-1}^{2}\left(\mu ; x_{i, m}(\mu)\right)\right| \sim & \left(1-x_{i, m}^{2}(\mu)\right)\left(\sqrt{1-x_{i, m}(\mu)}+m^{-1}\right)^{-2 \gamma-1} \\
& \times\left(\sqrt{1+x_{i, m}(\mu)}+m^{-1}\right)^{-2 \delta-1}
\end{aligned}
$$

uniformly for $-1 \leq x \leq 1$ and $m \in N$ [7, Theorem 9.30, p. 170].

Lemma 4.1 (Telyakovskii and Gopengauz). Let $f \in C^{q}$. Then for $n \geq 4 q+5$ there exists a sequence of polynomials $\left\{G_{n}\right\}$ such that for $|x| \leq 1$ and for $j=0,1, \ldots, q$

$$
\left|f^{(j)}(x)-G_{n}^{(j)}(x)\right| \leq \text { const }\left(\frac{\sqrt{1-x^{2}}}{n}\right)^{q-j} \omega\left(f^{(q)} ; \frac{\sqrt{1-x^{2}}}{n}\right),
$$

with some constant independent of $f$ and $n$. lemma.

Denoting by $r_{n}=f-G_{n}$ the remainder term, we can state the following

Lemma 4.2. Let $w, w_{1}, w_{2}, w_{3} \in \mathrm{GJ}$ be the weight functions defined by (2.8)(2.11). Let $L_{r}$ and $L_{s}$ be the polynomials defined by (2.5) and (2.6) with $Q_{n}=$ $p_{m+1}(w) p_{m}\left(w_{3}\right)$ or $Q_{n}=p_{m}\left(w_{1}\right) p_{m}\left(w_{2}\right)$. Then, for every function $f \in C^{q}$, $q \geq 0$, and $h \in\{0,1, \ldots, q\}$, there exist positive integers $r, s$, defined by

$$
\begin{aligned}
& \frac{h}{2}+\alpha+1 \leq r \leq \frac{h}{2}+\alpha+2, \\
& \frac{h}{2}+\beta+1 \leq s \leq \frac{h}{2}+\beta+2,
\end{aligned}
$$

such that

$$
\begin{aligned}
& \left|A_{s}(x) Q_{n}(x) L_{r}\left(Z ; \frac{r_{m}}{A_{s} Q_{n}} ; x\right)\right| \\
& \quad \leq \text { const }\left(\frac{\sqrt{1-x^{2}}}{m}+\frac{1}{m^{2}}\right)^{q} \omega\left(f^{(q)} ; \frac{\sqrt{1-x^{2}}}{m}+\frac{1}{m^{2}}\right),
\end{aligned}
$$




$$
\begin{aligned}
& \left|B_{r}(x) Q_{n}(x) L_{s}\left(Y ; \frac{r_{m}}{B_{r} Q_{n}} ; x\right)\right| \\
& \quad \leq \text { const }\left(\frac{\sqrt{1-x^{2}}}{m}+\frac{1}{m^{2}}\right)^{q} \omega\left(f^{(q)} ; \frac{\sqrt{1-x^{2}}}{m}+\frac{1}{m^{2}}\right),
\end{aligned}
$$

with some constants independent of $f$ and $m$.

Proof. In order to prove (4.9), we observe that by (4.5)

$$
p_{m+1}(w ; 1) p_{m}\left(w_{3} ; 1\right) \sim p_{m}\left(w_{1} ; 1\right) p_{m}\left(w_{2} ; 1\right) \sim m^{2 \alpha+2},
$$

and so

$$
\left|Q_{n}(x)\right| \sim m^{2 \alpha+2}, \quad z_{1, n} \leq x \leq 1 .
$$

Thus, by the Markov-Bernstein inequality,

$$
\left|Q_{n}^{\prime}(x)\right| \leq \text { const } m^{2}\left\|Q_{n}\right\|_{\left[z_{1, n}, 1\right]} \sim m^{2} Q_{n}(1), \quad z_{1, n} \leq x \leq 1,
$$

and

$$
\left|\left[\frac{1}{Q_{n}(x)}\right]^{\prime}\right|=\left|\frac{Q_{n}^{\prime}(x)}{Q_{n}^{2}(x)}\right| \sim \frac{m^{2}}{Q_{n}(1)}, \quad z_{1, n} \leq x \leq 1 .
$$

In view of the last inequality, and taking into account that

$$
\left[\frac{1}{Q_{n}(x)}\right]^{(l)}=\frac{1}{Q_{n}(x)} \sum_{j=0}^{l-1}\left(\begin{array}{l}
l \\
j
\end{array}\right)\left[\frac{1}{Q_{n}(x)}\right]^{(j)} Q_{n}^{(l-j)}(x)
$$

(see [7]), we deduce

$$
\left|\left[\frac{1}{Q_{n}(x)}\right]^{(l)}\right| \leq \text { const } \frac{m^{2 l}}{Q_{n}(1)}, \quad z_{1, n} \leq x \leq 1 .
$$

Now, we recall that

$$
\left[z_{1, n}, z_{2, n}, \ldots, z_{i, n} ; \frac{1}{A_{s} Q_{n}}\right]=\frac{1}{(i-1) !}\left[\frac{1}{A_{s}(x) Q_{n}(x)}\right]_{x=\xi_{i}}^{(i-1)},
$$

$z_{1, n} \leq \xi_{i} \leq z_{i, n}$, where the identity holds also when arbitrarily many $z_{j, n}$ coincide. So, by Leibniz' formula,

$$
\begin{aligned}
& \left|\left[z_{1, n}, z_{2, n}, \ldots, z_{i, n} ; \frac{1}{A_{s} Q_{n}}\right]\right| \\
& \quad \leq \frac{1}{(i-1) !} \sum_{l=0}^{i-1}\left(\begin{array}{c}
i-1 \\
l
\end{array}\right)\left|\left[\frac{1}{Q_{n}(x)}\right]_{x=\xi_{i}}^{(l)}\right|\left|\left[\frac{1}{A_{s}(x)}\right]_{x=\xi_{i}}^{(i-1-l)}\right| .
\end{aligned}
$$

Since the function $1 / A_{s}(x)$ and its derivatives are bounded for $x>0$, we get by $(4.11)$

$$
\left|\left[z_{1, n}, z_{2, n}, \ldots, z_{i, n} ; \frac{1}{A_{s} Q_{n}}\right]\right| \leq \text { const } \frac{1}{Q_{n}(1)} \sum_{l=0}^{i-1}\left(\begin{array}{c}
i-1 \\
l
\end{array}\right) m^{2 l} \sim \frac{m^{2 i-2}}{Q_{n}(1)} .
$$

Recalling the definition of $L_{r}\left(Z ; r_{m} /\left(A_{s} Q_{n}\right)\right)$, taking into account that $\left(x-z_{1, n}\right)\left(x-z_{2, n}\right) \cdots\left(x-z_{i-1, n}\right) \leq\left(\sqrt{1-x}+m^{-1}\right)^{2 i-2}$ for $|x| \leq 1$, and observing that by Lemma 4.1

$$
\left|r_{m}^{(k)}(x)\right| \leq \mathrm{const} m^{-2 q+2 k} \omega\left(f^{(q)} ; m^{-2}\right), \quad \begin{aligned}
& z_{1, m} \leq x \leq 1, k=0,1, \ldots, q, \\
& r \leq q+1,
\end{aligned}
$$


we deduce by Leibniz' formula that

$$
\left|L_{r}\left(Z ; \frac{r_{m}}{A_{s} Q_{n}} ; x\right)\right| \leq \mathrm{const} \frac{\omega\left(f^{(q)} ; m^{-2}\right)}{Q_{n}(1) m^{2 q}} \sum_{i=0}^{r-1}\left[(1-x) m^{2}+1\right]^{i},
$$

where $|x| \leq 1$. On the other hand, if $q=0$,

$$
L_{r}\left(Z ; \frac{r_{m}}{A_{s} Q_{n}} ; x\right)=\sum_{k=1}^{r} \prod_{\substack{i=1 \\ i \neq k}}^{r} \frac{x-z_{i, m}}{z_{k, m}-z_{i, m}} \frac{r_{m}\left(z_{k, m}\right)}{A_{s}\left(z_{k, m}\right) Q_{n}\left(z_{k, m}\right)} \text {. }
$$

Since

$$
\left|\prod_{\substack{i=1 \\ i \neq k}}^{r} \frac{x-z_{i, m}}{z_{k, m}-z_{i, m}}\right| \leq \operatorname{const}(m \sqrt{1-x}+1)^{2 r-2}, \quad|x| \leq 1,
$$

we again deduce (4.12). We first assume $|x| \leq 1-m^{-2}$; then by (4.12),

$$
\left|L_{r}\left(Z ; \frac{r_{m}}{A_{s} Q_{n}} ; x\right)\right| \leq \mathrm{const} \frac{\omega\left(f^{(q)} ; m^{-1} \sqrt{1-x^{2}}\right)}{Q_{n}(1) m^{2 q-2 r+2}}(1-x)^{r-1}
$$

Recalling that $Q_{n}(1) \sim m^{2 \alpha+2}$, we obtain

$$
\left|L_{r}\left(Z ; \frac{r_{m}}{A_{s} Q_{n}} ; x\right)\right| \leq \mathrm{const} \frac{\omega\left(f^{(q)} ; m^{-1} \sqrt{1-x^{2}}\right)}{m^{2(q-r+\alpha+2)}}(1-x)^{r-1},
$$

where $|x| \leq 1-m^{-2}$. The hypothesis $r \leq h / 2+\alpha+2$ assures that $q / 2-r+$ $\alpha+2 \geq 0$. Then, since $m^{-2} \leq 1-x^{2}$, we can write

$$
\begin{aligned}
\left|L_{r}\left(Z ; \frac{r_{m}}{A_{s} Q_{n}} ; x\right)\right| \leq & \text { const }\left(\frac{\sqrt{1-x^{2}}}{m}\right)^{q} \omega\left(f^{(q)} ; \frac{\sqrt{1-x^{2}}}{m}\right) \\
& \times(1-x)^{\alpha+1}(1+x)^{-r+\alpha+2}, \quad|x| \leq 1-m^{-2} .
\end{aligned}
$$

Since $\left|A_{s}(x)\right| \leq\left(\sqrt{1+x}+m^{-1}\right)^{2 s}$, and observing that by (4.4)

$$
\begin{array}{cc}
\left|p_{m+1}(w ; x) p_{m}\left(w_{3} ; x\right)\right| \leq \operatorname{const}(1-x)^{-\alpha-1}(1+x)^{-\beta-1}, & |x| \leq 1-m^{-2}, \\
\left|p_{m}\left(w_{1} ; x\right) p_{m}\left(w_{2} ; x\right)\right| \leq \operatorname{const}(1-x)^{-\alpha-1}(1+x)^{-\beta-1}, & |x| \leq 1-m^{-2},
\end{array}
$$

we have

$$
\begin{aligned}
\left|A_{s}(x) Q_{n}(x) L_{r}\left(Z ; \frac{r_{m}}{A_{s} Q_{n}} ; x\right)\right| \leq & \operatorname{const}\left(\frac{\sqrt{1-x^{2}}}{m}\right)^{q} \omega\left(f^{(q)} ; \frac{\sqrt{1-x^{2}}}{m}\right) \\
& \times(1+x)^{s-r+\alpha-\beta+1}, \quad|x| \leq 1-m^{-2} .
\end{aligned}
$$

From this last inequality, (4.9) follows immediately for $0 \leq x \leq 1-m^{-2}$. On the other hand, if $-1+m^{-2} \leq x<0$, it is sufficient to observe that the assumptions $s \geq h / 2+\beta+1$ and $r \leq h / 2+\alpha+2$ assure that $s-r+\alpha-\beta+1 \geq 0$. Finally, if $|x| \geq 1-m^{-2}$, then inequality (4.9) follows immediately by (4.12). Similarly, one can prove inequality (4.10).

We omit the proof of the following lemma, since it is very similar to that of Lemma 4.2. 
Lemma 4.3. Let $w \in \mathrm{GJ}$ be the weight function defined by (2.8). Let $L_{r}$ and $L_{s}$ be the polynomials defined by (2.5) and (2.6) with $Q_{n}=p_{m}(w) p_{m+1}(w)$. Then, for every function $f \in C^{q}, q \geq 0$, and $h \in\{0,1, \ldots, q\}$, there exist positive integers $r, s$, defined by

$$
\frac{h}{2}+\alpha \leq r \leq \frac{h}{2}+\alpha+\frac{3}{2}, \quad \frac{h}{2}+\beta \leq s \leq \frac{h}{2}+\beta+\frac{3}{2},
$$

such that

$$
\begin{aligned}
& \left|A_{s}(x) Q_{n}(x) L_{r}\left(Z ; \frac{r_{m}}{A_{s} Q_{n}} ; x\right)\left(\sqrt{1-x^{2}}+\frac{1}{m}\right)\right| \\
& \leq \text { const }\left(\frac{\sqrt{1-x^{2}}}{m}+\frac{1}{m^{2}}\right)^{q} \omega\left(f^{(q)} ; \frac{\sqrt{1-x^{2}}}{m}+\frac{1}{m^{2}}\right) \text {, } \\
& \left|B_{r}(x) Q_{n}(x) L_{s}\left(Y ; \frac{r_{m}}{B_{r} Q_{n}} ; x\right)\left(\sqrt{1-x^{2}}+\frac{1}{m}\right)\right| \\
& \leq \text { const }\left(\frac{\sqrt{1-x^{2}}}{m}+\frac{1}{m^{2}}\right)^{q} \omega\left(f^{(q)} ; \frac{\sqrt{1-x^{2}}}{m}+\frac{1}{m^{2}}\right) \text {, }
\end{aligned}
$$

with some constants independent of $f$ and $m$.

Later we need the following result. For any weight function $\mu \in \mathrm{GJ}$ and for every $x \in[-1,1]$, we have

$$
\begin{array}{r}
\sum_{\substack{k=1 \\
k \neq c}}^{m} \frac{\left(1-x_{k, m}(\mu)\right)^{\rho}}{m\left|x-x_{k, m}(\mu)\right|} \leq \operatorname{const}\left(\sqrt{1-x}+m^{-1}\right)^{2 \rho-1} \log m \\
\text { if }-\frac{1}{2} \leq \rho \leq \frac{1}{2}, \\
\sum_{\substack{k=1 \\
k \neq c}}^{m} \frac{\left(1+x_{k, m}(\mu)\right)^{\sigma}}{m\left|x-x_{k, m}(\mu)\right|} \leq \operatorname{const}\left(\sqrt{1+x}+m^{-1}\right)^{2 \sigma-1} \log m \\
\text { if }-\frac{1}{2} \leq \sigma \leq \frac{1}{2},
\end{array}
$$

where $c$ denotes the index corresponding to the closest knot(s) to $x$, and $\rho, \sigma$ are real numbers. The proof of these inequalities can be found in [3].

Now we prove the theorems stated in the previous section.

Proof of Theorem 3.1. We start with the case $h=0$ in (3.1). Let $r_{m}=f-G_{m}$, where $G_{m}$ is the polynomial defined by Lemma 4.1. Then, (4.17) $\left|f(x)-L_{2 m+1, r, s}\left(w, w_{3} ; f ; x\right)\right| \leq\left|r_{m}(x)\right|+\left|L_{2 m+1, r, s}\left(w, w_{3} ; r_{m} ; x\right)\right|$. Recalling (2.12), and applying Lemma 4.2 with $h=l$, we find

$$
\begin{aligned}
\mid L_{2 m+1, r, s}(w, & \left.w_{3} ; r_{m} ; x\right) \mid \\
\leq \text { const }\{ & \left(\frac{\sqrt{1-x^{2}}}{m}+\frac{1}{m^{2}}\right)^{q} \omega\left(f^{(q)} ; \frac{\sqrt{1-x^{2}}}{m}+\frac{1}{m^{2}}\right) \\
& +\left|A_{s}(x) B_{r}(x) p_{m+1}(w ; x) p_{m}\left(w_{3} ; x\right)\right| \\
& \left.\times\left[\left|H_{m}\left(w_{3} ; \frac{r_{m}}{A_{s} B_{r}} ; x\right)\right|+\left|H_{m+1}\left(w ; v^{1,1} \frac{r_{m}}{A_{s} B_{r}} ; x\right)\right|\right]\right\} .
\end{aligned}
$$


By

$$
\left|A_{s}(x) B_{r}(x)\right| \leq\left(\sqrt{1+x}+m^{-1}\right)^{2 s}\left(\sqrt{1-x}+m^{-1}\right)^{2 r}, \quad|x| \leq 1,
$$

and Lemma 4.1, we obtain

$$
\begin{aligned}
\left|L_{2 m+1, r, s}\left(w, w_{3} ; r_{m} ; x\right)\right| & \\
\leq & \operatorname{const} \frac{\omega\left(f^{(q)} ; m^{-1}\right)}{m^{q}}\left|A_{s}(x) B_{r}(x) p_{m+1}(w ; x) p_{m}\left(w_{3} ; x\right)\right| \\
& \times\left\{\left|H_{m}\left(w_{3} ; v^{q / 2-r, q / 2-s} ; x\right)\right|+\left|H_{m+1}\left(w ; v^{q / 2-r+1, q / 2-s+1} ; x\right)\right|\right\} \\
= & : \Sigma_{1}+\Sigma_{2} .
\end{aligned}
$$

As above, let $c$ be the index of the knots $x_{i, m}\left(w_{3}\right)$ corresponding to the closest $\operatorname{knot}(\mathrm{s})$ to $x$. Then, inequalities (4.3), (4.4), and (4.7) allow us to write

$$
\left|p_{m}\left(w_{3} ; x\right) p_{m+1}(w ; x)\right| \frac{\lambda_{c, m}\left(w_{3}\right)}{\left|x-x_{c, m}\left(w_{3}\right)\right|} \sim 1 \text {. }
$$

Thus, applying again inequalities (4.3) and (4.4), we deduce

$$
\begin{aligned}
\Sigma_{1} \leq & \text { const } \frac{\omega\left(f^{(q)} ; m^{-1}\right)}{m^{q}}\left(\sqrt{1-x}+m^{-1}\right)^{2(r-\alpha-1)} \\
& \times\left(\sqrt{1+x}+m^{-1}\right)^{2(s-\beta-1)} \\
& \times \sum_{i \neq c} \frac{v^{q / 2-r+\alpha+3 / 2, q / 2-s+\beta+3 / 2}\left(x_{i, m}\left(w_{3}\right)\right)}{m\left|x-x_{i, m}\left(w_{3}\right)\right|} \\
\leq & \text { const } \frac{\omega\left(f^{(q)} ; m^{-1}\right)}{m^{q}}\left(\sqrt{1-x}+m^{-1}\right)^{2(r-\alpha-1)} \\
& \times\left(\sqrt{1+x}+m^{-1}\right)^{2(s-\beta-1)} \\
& \times \sum_{i \neq c} \frac{v^{l / 2-r+\alpha+3 / 2, l / 2-s+\beta+3 / 2}\left(x_{i, m}\left(w_{3}\right)\right)}{m\left|x-x_{i, m}\left(w_{3}\right)\right|}
\end{aligned}
$$

for every $l \in\{0,1, \ldots, q\}$. Since $l / 2-r+\alpha+1 \leq 0$ and $l / 2-s+\beta+1 \leq 0$, we have

$$
\begin{aligned}
\Sigma_{1} \leq & \text { const } \frac{\omega\left(f^{(q)} ; m^{-1}\right)}{m^{q}}\left(\sqrt{1-x}+m^{-1}\right)^{2(r-\alpha-1)} \\
& \times\left(\sqrt{1+x}+m^{-1}\right)^{2(s-\beta-1)} \\
& \times\left\{\left(\sqrt{1-x}+m^{-1}\right)^{l-2 r+2 \alpha+2} \sum_{i<c} \frac{\left(1+x_{i, m}\left(w_{3}\right)\right)^{l / 2-s+\beta+3 / 2}}{m\left(x-x_{i, m}\left(w_{3}\right)\right)}\right. \\
& \left.+\left(\sqrt{1+x}+m^{-1}\right)^{l-2 s+2 \beta+2} \sum_{i>c} \frac{\left(1-x_{i, m}\left(w_{3}\right)\right)^{l / 2-r+\alpha+3 / 2}}{m\left(x_{i, m}\left(w_{3}\right)-x\right)}\right\} .
\end{aligned}
$$

By (3.2) and (3.3) we have $-\frac{1}{2}<l / 2-s+\beta+3 / 2 \leq 1 / 2$ and $-1 / 2<l / 2-r+\alpha+$ $3 / 2 \leq 1 / 2$; whence (4.15) and (4.16) can be applied with $\rho=l / 2-r+\alpha+3 / 2$ and $\sigma=l / 2-s+\beta+3 / 2$, respectively. We get

$$
\Sigma_{1} \leq \mathrm{const} \frac{\omega\left(f^{(q)} ; m^{-1}\right)}{m^{q}}\left(\sqrt{1-x^{2}}+m^{-1}\right)^{l} \log m .
$$


Similarly,

$$
\Sigma_{2} \leq \mathrm{const} \frac{\omega\left(f^{(q)} ; m^{-1}\right)}{m^{q}}\left(\sqrt{1-x^{2}}+m^{-1}\right)^{l} \log m .
$$

These relations, together with (4.18), give us

$$
\begin{aligned}
& \left|L_{2 m+1, r, s}\left(w, w_{3} ; r_{m} ; x\right)\right| \\
& \quad \leq \text { const }\left[\frac{\sqrt{1-x^{2}}}{m}+\frac{1}{m^{2}}\right]^{l} \omega\left(f^{(q)} ; \frac{1}{m}\right) \frac{\log m}{m^{q-l}} .
\end{aligned}
$$

Then, by (4.17), and in view of Lemma 4.1, we deduce the first inequality in (3.1) for $h=0$. In order to prove (3.1) for $1 \leq h \leq l$, we recall that if $R_{n}$ is an algebraic polynomial of degree $n$ such that

$$
\left|R_{n}(x)\right| \leq \text { const }\left(\frac{\sqrt{1-x^{2}}}{n}+\frac{1}{n^{2}}\right)^{\nu}, \quad|x| \leq 1,
$$

where $\nu$ is a real number, then for any integer $j$

$$
\left|R_{n}^{(j)}(x)\right| \leq \text { const }\left(\frac{\sqrt{1-x^{2}}}{n}+\frac{1}{n^{2}}\right)^{\nu-j}, \quad|x| \leq 1,
$$

for some constant independent of $\nu$ and $j$. (See [4].) So, by (4.20), we can write

$$
\left|L_{2 m+1, r, s}^{(h)}\left(w, w_{3} ; r_{m} ; x\right)\right| \leq \text { const }\left[\frac{\sqrt{1-x^{2}}}{m}+\frac{1}{m^{2}}\right]^{l-h} \omega\left(f^{(q)} ; \frac{1}{m}\right) \frac{\log m}{m^{q-l}} .
$$

Therefore,

$$
\left|f^{(h)}(x)-L_{2 m+1, r, s}^{(h)}\left(w, w_{3} ; f ; x\right)\right| \leq\left|r_{m}^{(h)}(x)\right|+\left|L_{2 m+1, r, s}^{(h)}\left(w, w_{3} ; r_{m} ; x\right)\right| .
$$

Finally, estimating $\left|r_{m}^{(h)}(x)\right|$ by Lemma 4.1 , we deduce the first inequality of (3.1) also for $1 \leq h \leq l$. The proof of the second inequality of (3.1) is similar.

Proof of Theorem 3.3. We first observe that by (3.11) we have $A_{s} B_{r}=v^{r, s}$. Now, let $r_{m}=f-G_{m}$, where $G_{m}$ is the polynomial of Lemma 4.1; then $r_{m}^{(k)}( \pm 1)=0, k=0,1, \ldots, q$. Therefore, recalling (2.14), since $r, s \leq q+1$, we find

$$
\begin{aligned}
& \left|L_{2 m+1, r, s}\left(w, w_{3} ; r_{m} ; x\right)\right| \\
& =\operatorname{const} v^{r, s}(x)\left|p_{m+1}(w ; x) p_{m}\left(w_{3} ; x\right)\right| \\
& \quad \times\left\{\left\|H_{m}\left(w_{3} ; \frac{r_{m}}{v^{r, s}} ; x\right)\right\|+\left\|H_{m+1}\left(w ; \frac{v^{1,1} r_{m}}{v^{r, s}} ; x\right)\right\|\right\} \\
& =: \operatorname{const}\left\{S_{1}+S_{2}\right\} .
\end{aligned}
$$

If we assume $x_{m, 1}(w) \leq x \leq x_{m, m}(w)$, then we can proceed as in the proof of Theorem 3.1. Indeed, in this case, $\sqrt{1-x^{2}}>m^{-1}$, and by (4.19) we have

$$
L_{2 m+1, r, s}\left(w, w_{3} ; r_{m} ; x\right) \leq \text { const }\left(\frac{\sqrt{1-x^{2}}}{m}\right)^{l} \omega\left(f^{(q)} ; \frac{1}{m}\right) \frac{\log m}{m^{q-l}},
$$

for $x_{m, 1}(w) \leq x \leq x_{m, m}(w)$ 
On the other hand, if $x_{m, m}(w)<x<1$, then by Lemma 4.1, and in view of the inequalities (4.3), (4.5), and (4.19),

$$
\begin{aligned}
& S_{1} \leq \text { const }\left\{\frac{v^{r, s}(x)}{m^{q}} \omega\left(f^{(q)} ; \frac{1}{m}\right) m^{2 \alpha+2} \sum_{i=1}^{m-2} \frac{v^{q / 2-r+\alpha+3 / 2, q / 2-s+\beta+3 / 2}\left(x_{m, i}\left(w_{1}\right)\right)}{m\left(x-x_{m, i}\left(w_{1}\right)\right)}\right. \\
& \leq \text { const }\left\{\frac{v^{r, s}(x)}{m^{q-2-2 \alpha}} \omega\left(f^{(q)} ; \frac{1}{m}\right) \sum_{i=1}^{m-2} \frac{v^{l / 2-r+\alpha+3 / 2, l / 2-s+\beta+3 / 2}\left(x_{m, i}\left(w_{1}\right)\right)}{m\left(x-x_{m, i}\left(w_{1}\right)\right)}\right. \\
&\left.+\left(\frac{\sqrt{1-x^{2}}}{m}\right)^{q} \omega\left(f^{(q)} ; \frac{1}{m}\right)\right\} \\
& \leq \text { const }\left\{\frac{v^{r, s}(x)}{\left.m^{q-2-2 \alpha} \omega\left(f^{(q)} ; \frac{1}{m}\right)\right\}}\right. \\
& \times \int_{-1}^{x_{m, m-1}(w)}(1-u)^{l / 2-r+\alpha+1} \frac{(1+u)^{l / 2-s+\beta+1}}{x-u} d u \\
&\left.+\left(\frac{\sqrt{1-x^{2}}}{m}\right)^{q} \omega\left(f^{(q)} ; \frac{1}{m}\right)\right\} .
\end{aligned}
$$

The last integral can be easily estimated. Indeed, since $l / 2-s+\beta+1>-1$,

$$
\begin{aligned}
& \int_{-1}^{x_{m, m-1}(w)}(1-u)^{l / 2-r+\alpha+1} \frac{(1+u)^{l / 2-s+\beta+1}}{x-u} d u \\
& \quad \leq\left\{\int_{-1}^{0}+\int_{0}^{x_{m, m-1}(w)}\right\}(1-u)^{l / 2-r+\alpha+1} \frac{(1+u)^{l / 2-s+\beta+1}}{x-u} d u \\
& \quad \leq \operatorname{const}\left\{1+(1-x)^{l / 2-r+\alpha+1} \int_{\left(1-x_{m, m-1}(w)\right) /(1-x)}^{1 /(1-x)} \frac{y^{l / 2-r+\alpha+1}}{y-1} d y\right\} .
\end{aligned}
$$

Further, since $-1<l / 2-r+\alpha+1 \leq 0$ and $y>1$, we can write

$$
\begin{aligned}
& \int_{-1}^{x_{m, m-1}(w)}(1-u)^{l / 2-r+\alpha+1} \frac{(1+u)^{l / 2-s+\beta+1}}{x-u} d u \\
& \leq \operatorname{const}(1-x)^{l / 2-r+\alpha+1} \log m .
\end{aligned}
$$

Therefore, (4.21) is still valid when $x_{m, m}(w)<x<1$, and similarly for $-1<$ $x<x_{m, 1}(w)$. Then, by (4.17) and Lemma 4.1, we deduce the first inequality of $(3.12)$ for $h=0$. Proceeding as in the last part of the proof of Theorem 3.1 , we can obtain the first inequality of $(3.12)$ also for $1 \leq h \leq l$. The proof of the second inequality of (3.12) is similar.

The proofs of Theorems 3.2 and 3.4 are analogous to those of Theorems 3.1 and 3.3, respectively. We omit the details. 
Proof of Theorems 3.5 and 3.7. Proceeding as in the proof of Theorem 3.1, we find

$$
\begin{aligned}
\left|L_{2 m+1, r, s}\left(w, w_{3} ; r_{m} ; x\right)\right| \leq & \frac{\text { const }}{m^{q+\lambda}}\left|A_{s}(x) B_{r}(x) p_{m+1}(w ; x) p_{m}\left(w_{3} ; x\right)\right| \\
& \times\left\{\left|H_{m}\left(w_{3} ; v^{q / 2+\lambda / 2-r, q / 2+\lambda / 2-s} ; x\right)\right|\right. \\
& \left.\quad+\left|H_{m+1}\left(w ; v^{q / 2+\lambda / 2-r+1, q / 2+\lambda / 2-s+1} ; x\right)\right|\right\},
\end{aligned}
$$

instead of (4.18). Then, by the same steps as in the proof of Theorem 3.1, we obtain the first inequality of (3.16). The second one can be proved analogously.

Furthermore, if the additional knots satisfy the relation (3.11), then from (4.21) and by Lemma 4.1,

$$
\begin{aligned}
\left|L_{2 m+1, r, s}\left(w, w_{3} ; r_{m} ; x\right)\right| \leq & \frac{\text { const }}{m^{q+\lambda}} v^{r, s}(x)\left|p_{m+1}(w ; x) p_{m}\left(w_{3} ; x\right)\right| \\
& \times\left\{\left|H_{m}\left(w_{3} ; v^{\lambda / 2-r, \lambda / 2-s} ; x\right)\right|\right. \\
& \left.\quad+\left|H_{m+1}\left(w ; v^{\lambda / 2-r+1, \lambda / 2-s+1} ; x\right)\right|\right\},
\end{aligned}
$$

and proceeding as in the proof of Theorem 3.3, we deduce the first (or second) inequality of (3.22).

Theorems 3.6 and 3.8 can be proved similarly as Theorems 3.5 and 3.7 , respectively. We omit the details.

\section{BIBLIOGRAPHY}

1. V. M. Badkov, Convergence in the mean and almost everywhere of Fourier series in polynomials orthogonal on an interval, Math. USSR-Sb. 24 (1974), 223-256.

2. G. Criscuolo, G. Mastroianni, and D. Occorsio, Convergence of extended Lagrange interpolation, Math. Comp. 55 (1990), 197-212.

3. G. Criscuolo and G. Mastroianni, Mean and uniform convergence of quadrature rules for evaluating the finite Hilbert transform, Progress in Approximation Theory (P. Nevai and A. Pinkus, eds.), Academic Press, 1991, pp. 141-176.

4. V. K. Dzjadik, Introduction to uniform approximation of functions by polynomials, Nauka, Moscow, 1977. (Russian)

5. W. Gautschi, Gauss-Kronrod quadrature-A survey, Numerical Methods and Approximation Theory III (G. V. Milovanović, ed.), Prosveta, Niš, 1988, pp. 39-66.

6. G. Monegato, Stieltjes polynomials and related quadrature rules, SIAM Rev. 24 (1982), 137-158.

7. P. Nevai, Orthogonal polynomials, Mem. Amer. Math. Soc., No. 213 (1979).

8. P. Nevai and P. Vértesi, Mean convergence of Hermite-Fejér interpolation, J. Math. Anal. Appl. 105 (1985), 26-58.

(G. Criscuolo and G. Mastroianni) Istituto Per Applicazioni della Matematica, C.N.R., Via P. Castellino 111, 80131 Napoli, Italy

E-mail address, G. Criscuolo: iam@iamna.na.cnr.it

(G. Mastroianni) Istituto di Matematica, Università della Basilicata, Via N. Sauro 85, 85100 Potenza, Italy

(P. Vértesi) Mathematical Institute of the Hungarian Academy of Sciences, 1053 BuDAPEST, Reáltanoda U.13-15, Hungary 